\title{
Endo-irreversible thermo-mechanical Carnot engine with new concept of entropy production action coefficient
}

\author{
Michel Feidt ${ }^{1, *}$ and Renaud Feidt ${ }^{2}$ \\ ${ }^{1}$ Université de Lorraine, CNRS, LEMTA, Nancy F-54000, France \\ ${ }^{2}$ SAS INVIVO Consulting, Nantes F-44000, France
}

Received: 30 December 2020 / Received in final form: 28 March 2021 / Accepted: 21 May 2021

\begin{abstract}
Thermostatics of Carnot engines has been extended by more recent research based on endoreversible model. Our model assumes exo-reversibility but endo-irreversibility to determine new upper-bound to thermomechanical conversion. We propose a functional expression of entropy production related to cycle transformation durations. This approach analyses the energy, entropy and power consequences. We introduce a new concept of entropy production actions that results in three sequential optimums: maximum energy related to transformation durations, maximum energy associated with equipartition of entropy actions, optimal power for given cycle period.
\end{abstract}

\section{Introduction}

The industrial revolution took off in conjunction with that of Thermodynamics. While the engineer's concerns were important, the fact remains that more fundamental research has accompanied the development of thermal engines (or more precisely thermo-mechanics).

Under the probable influence of his father L. Carnot who was a scientist and a mathematician [1], S. Carnot was the first to make the link between mechanics and thermal science [2] in a determining contribution that makes him one of the founding fathers of the Equilibrium Thermodynamics. He is thus credited with several decisive contributions [3]:

- the notion of thermodynamic cycle, including the cycle that bears its name.

- the extension of the notion of mechanical efficiency, to the efficiency of a thermomechanical engine (we now say internal efficiency in the sense of the first law of thermodynamics, $\eta_{I}$ ), adimensional form of efficiency.

S. Carnot has shown that this efficiency is an upper bound that is called, the Carnot efficiency, $\eta_{C}$. This efficiency characterize the two heat reservoirs engines, operating almost statically (Thermodynamics of equilibrium, T.E.). Assuming the isothermal source at $\mathrm{T}_{\mathrm{HS}}$ (thermostat), as well as the isothermal sink at $\mathrm{T}_{\mathrm{CS}}<\mathrm{T}_{\mathrm{HS}}$, he obtains:

$$
\eta_{C}=1-\frac{T_{C S}}{T_{H S}}
$$

$T_{C S}$, cold sink temperature; $T_{H S}$, hot source temperature.

\footnotetext{
* e-mail: michel.feidt@univ-lorraine.fr
}

Since then, numerous studies have been carried out on the Carnot cycle, or the Carnot engines (268 references with these keywords have been recorded for the last 3 years). The scope of this list goes far beyond engineering and classical physics, to quantum, molecular, photonic motors... Other models leave the small scales (nanophysics) to consider large structures (especially black holes), or life.

For this proposal, we will continue to focus on the phenomenological approach of classical thermodynamics at mesoscopic (human) scales.

In this context, there is a revival of work aimed at considering the influence of out-of-balance cycles transformations. The article most often cited as marking this revival is the 1975 article by Curzon-Ahlborn [4]. In fact, as we have shown [5], this work was preceded by other work in $1957[6,7]$ and even before [8].

The majority of these works assumes temperature differences between the hot source and the engine (possibly between the engine and the sink), but in the end, considers an endo-reversible engine (without internal irreversibility) for optimization.

In addition, the optimization focuses on $(\operatorname{MAX} \dot{W})$ maximum power (in watt) of the engine. The choice of this objective function is logical, since it is the useful effect of an engine. Nevertheless, some details will be added in this article. The key result of the optimization is the demonstration of the engine's performance at maximum power:

$$
\eta_{I}(\mathrm{MAX} \dot{W})=1-\sqrt{\frac{T_{C S}}{T_{H S}}} .
$$


This efficiency is much lower than the efficiency of Carnot, but the engine provides a given power (which is not the case for the Equilibrium Thermodynamics model). Since then, research has shown that the relationship (2) is not generic [9-11]. This article essentially proposes two things:

- unlike the majority of proposals that focus on external heat transfers irreversibilities, we develop the case where heat transfers are perfect (exo-reversibility), but where the internal irreversibilities of the converter are taken into account whatever they are. Low dissipation models [11] or pseudo-cycles of Carnot [12] are not used. Then the importance of heat transfer entropies appears (see Sect. 2).

- we will consider 2 major cases of optimization: either in W energy or in average power $\dot{W}$ (and not instantaneous power, as we will justify (see Sect. 3 ).

- in these cases, the influence of the form of internal irreversibilities will be particularly studied, as well as the consequences resulting from it.

Section 4 will be dedicated to the discussion and synthesis of the results. The resulting perspectives will be proposed.

\section{Endo-irreversible model for Carnot engine}

Unlike the vast majority of articles on the subject, we present a model whose irreversibilities are internal to the converter and represented globally, as will be seen below. Section 3 is relative to power optimization contrary to Section 2 concerned by energy optimization.

\subsection{Models with thermal losses}

However, to be complete, Figure 1 shows the Carnot engine between the hot thermostat at $\mathrm{T}_{\mathrm{HS}}$ and the cold thermostat at $\mathrm{T}_{\mathrm{CS}}$. It is noted that this diagram incorporates the presence of thermal losses (through the material structure) between the warmest point and the coldest point. The heat loss conductance equivalent to the $\mathrm{K}_{\mathrm{LS}}$ system typically leads, assuming linear heat transfers, to the expression of the lost heat energy $Q_{\mathrm{LS}}(\mathrm{J})$ :

$$
Q_{L S}=K_{L S} \times \zeta \times\left(T_{H S}-T_{C S}\right)
$$

$\zeta$, operating time to be specified (s).

$K_{L S}$, average conductance of heat losses over the operating time $(\mathrm{W} / \mathrm{K})$.

Generally speaking, the following will be written:

$$
Q_{L S}=G_{L S} \times\left(T_{H S}-T_{C S}\right)=T_{H S} \times \Delta S_{L S}
$$

$G_{L S}$, energy conductance of thermal losses $\left(\mathrm{J}^{\mathrm{K}} \mathrm{K}^{-1}\right)$

$\Delta S_{L S}$, heat loss transfer entropy, (J.K $\left.{ }^{-1}\right)$ at the source during $\zeta$.

To preserve generality as much as possible, we will keep the $Q_{H S}$ energy expenditure in the form of:

$$
Q_{H S}=Q_{H}+Q_{L S}
$$

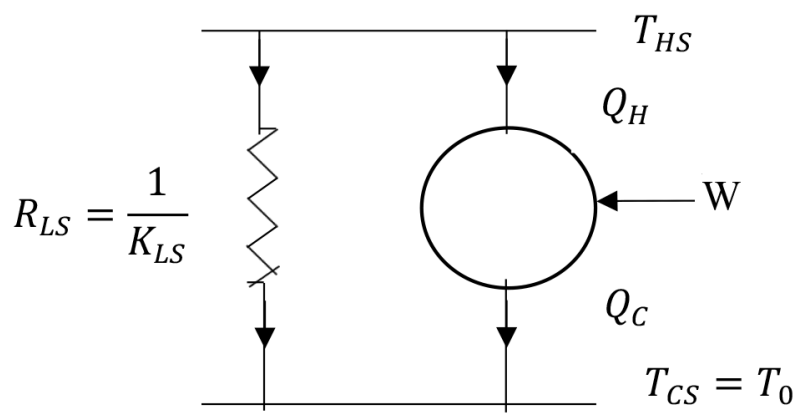

Fig. 1. General schematic representation of the endo-irreversible Carnot engine with thermal losses.

By posing $Q_{H}=T_{H S} \times \Delta S_{H}$, as well as $Q_{C}=T_{C S} \times \Delta S_{C}$, we get:

$$
Q_{H S}=T_{H S} \times\left(\Delta S_{H}+\Delta S_{L S}\right) .
$$

The entropy balance to the converter is written over the same period:

$$
\Delta S_{C}=\Delta S_{H}+\Delta S_{I}
$$

$\Delta S_{I}$, internal entropy production of converter during $\zeta$.

We deduce, the mechanical work produced over the same period, using the energy balance of the converter by difference of heat at the hot source, then at the cold sink of the engine $\left(Q_{H}-Q_{C}\right)$ :

$$
W=\left(T_{H S}-T_{C S}\right) \times \Delta S_{H}-T_{C S} \times \Delta S_{I} .
$$

We find here that the maximum mechanical energy is obtained when the production of entropy is cancelled (totally reversible cycle). But we note that this energy depends on the thermal potential $\Delta T_{S}=T_{H S}-T_{0}$, and also on the converted entropy of heat transfer between hot thermostat and converter, which we will take as reference $\Delta S_{H}=\Delta S$.

The expression of performance in the sense of the first law of thermodynamics is then expressed by:

$$
\begin{gathered}
\eta_{I}=\frac{W}{Q_{H S}}=\frac{\left(T_{H S}-T_{C S}\right) \times \Delta S-T_{C S} \times \Delta S_{I}}{T_{H S} \times\left(\Delta S+\Delta S_{L S}\right)} \\
\eta_{I}=\frac{1}{1+d_{L S}} \times\left[1-\frac{T_{C S}}{T_{H S}} \times\left(1+d_{I}\right)\right]
\end{gathered}
$$

or

$$
\eta_{I}=\left[\eta_{C}-\left(1-\eta_{C}\right) \times d_{I}\right] \times \frac{1}{1+d_{L S}}
$$

with $d_{I}$, degree of irreversibility of the converter $=\frac{\Delta S_{I}}{\Delta S}$

$d_{L S}$, degree of thermal loss (for the system including source and sink $)=\frac{\Delta S_{L S}}{\Delta S}$.

The expression $(9)$ shows that heat losses introduce a reduction in efficiency related to the ratio $d_{L S}$ (usually in the order of a few percent). Similarly, internal irreversibilities 
attenuate the efficiency through $d_{I}$, but weighted by the factor $\frac{\left(1-\eta_{C}\right)}{\eta_{C}}$ as can be seen on (9ter). Engines with a small temperature difference are therefore penalized.

Moreover, the existence of a source temperature threshold leading to a performance that is physically acceptable to the engine can be observed with a fixed degree of irreversibility:

$$
\eta_{C}>\frac{d_{I}}{1+d_{I}} .
$$

In general, $d_{I}$ is less than 1 .

Or at given temperatures, the existence of an irreversibility threshold:

$$
d_{I}<\frac{\eta_{C}}{1-\eta_{C}}
$$

The study related to the degree of thermal loss of the system only makes sense for a heat thermostat of finite energy $Q_{0}$. It is then easy to show that:

$$
\Delta S=\frac{Q_{0}-T_{H S} \times \Delta S_{L S}}{T_{H S}} \geq 0 .
$$

This leads to either a condition on $\Delta S$

$$
\Delta S \times\left(1+d_{L S}\right) \leq \frac{Q_{0}}{T_{H S}} .
$$

Either on a condition of $Q_{0}$

$$
Q_{0} \geq T_{H S} \times \Delta S_{L S}
$$

But the relationship (10) also shows the existence of a maximum temperature achievable for a finite energy source, or temperature of stagnation $T_{S}$ (common concept in solar energy)

$$
T_{H S}<\frac{Q_{0}}{d_{L S} \times \Delta S}=T_{S} .
$$

This relationship can also be interpreted in 2 other forms:

or

$$
\Delta S<\frac{Q_{0}}{T_{H S} \times d_{L S}}
$$

$$
d_{L S}<\frac{Q_{0}}{T_{H S} \times \Delta S} .
$$

They induce, at $T_{H S}$ temperature and source energy imposed, either a limitation of transfer entropy to the converter, or a limitation of the degree of thermal losses.

In the following paragraph, we will assume for simplicity, an endo-irreversible Carnot engine without thermal losses.

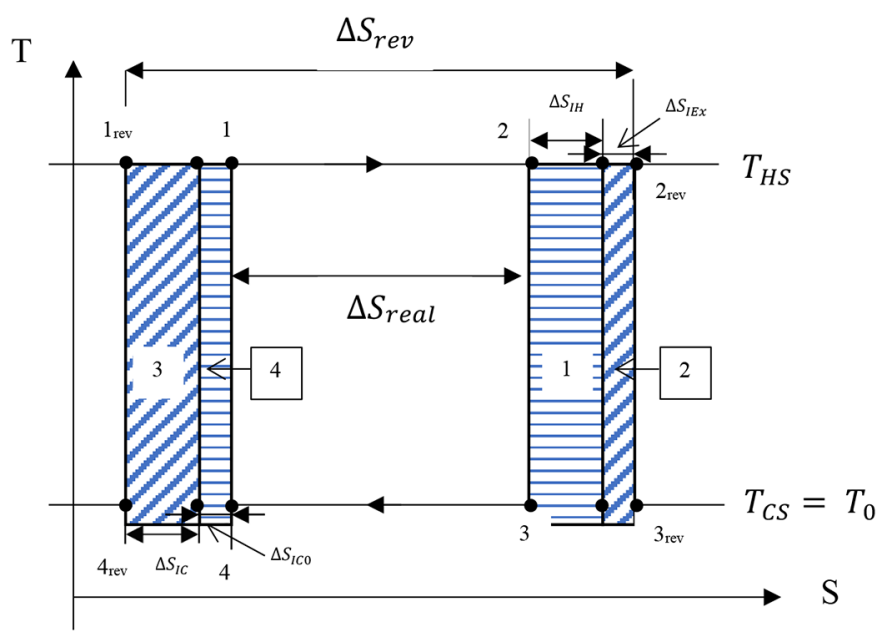

Fig. 2. Representation of the reversible and endo-irreversible Carnot cycles, in the diagram $(\mathrm{T}, \mathrm{S})$.

\subsection{Endo-irreversible Carnot engine model}

The endo-irreversible Carnot cycle is shown in Figure 2. It typically consists of four transformations: 2 isotherms and 2 irreversible adiabatics. The fully reversible cycle is shown in Figure 2 as cycle 1 rev -2 rev -3 rev -4 rev. It corresponds to the input of the converter $Q_{H \text { rev }}$ as:

$$
Q_{H \text { rev }}=T_{H S} \times \Delta S_{H r e v} .
$$

For this same reversible cycle, but at the cold end, we obtain:

$$
Q_{C r e v}=T_{C S} \times \Delta S_{C r e v}=T_{C S} \times \Delta S_{H r e v} .
$$

The mechanical energy in the reversible case is then expressed in the form:

$$
\begin{aligned}
W_{\text {rev }} & =Q_{H \text { rev }}-Q_{C r e v}=\left(T_{H S}-T_{C S}\right) \times \Delta S_{H \text { rev }} \\
& =\Delta T_{S} \times \Delta S_{H \text { rev }} .
\end{aligned}
$$

For the sake of simplification, the following are provided (see Fig. 2):

$$
\Delta S_{\text {Hrev }}=\Delta S_{\text {Crev }}=\Delta S_{\text {rev }} .
$$

For the endo-irreversible cycle, at each transformation a production of entropy is associated: $\Delta S_{I H}$ for the high temperature isotherm; $\Delta S_{I E x}$ for the adiabatic expansion; $\Delta S_{I C}$ for the low temperature isotherm; $\Delta S_{I C 0}$ for the adiabatic compression.

Each transformation degrades mechanical energy = area 1 for the high temperature isotherm; area 2 for the adiabatic expansion, area 3 for the low temperature isotherm; area 4 for the adiabatic compression. Therefore, the mechanical work of the endo-irreversible cycle corresponds to area 1-2-3-4:

$$
W_{\text {real }}=\left(T_{H S}-T_{C S}\right) \times \Delta S_{\text {real }} .
$$


However, the entropic balance of the cycle (S, state function) provides:

$$
\begin{aligned}
\Delta S_{S} & =\Delta S_{\text {rev }}=\Delta S_{\text {real }}+\Delta S_{I H}+\Delta S_{I C}+\Delta S_{I E x}+\Delta S_{I C 0} \\
& =\Delta S_{\text {real }}+\Delta S_{I}
\end{aligned}
$$

where:

$$
W_{\text {real }}=\left(T_{H S}-T_{C S}\right) \times\left(\Delta S_{S}-\Delta S_{I}\right)
$$

$\Delta S_{S}$, heat transfer entropy available at the source in the reversible case, or total entropy of the system (source + converter + sink)

$\Delta S_{I}$, total entropy production of the cycle.

$\Delta S_{\text {real }}$ actually, corresponds to conversion entropy. We deduce the expression of the efficiency of the endoirreversible motor in relation to the heat transfer entropy of the reversible case $\Delta S_{S}=\Delta S_{r e v}$ :

$$
\eta_{I}=\eta_{C} \times\left(1-d_{I}\right)
$$

with $d_{I}=\frac{\Delta S_{I}}{\Delta S_{S}}$, the degree of irreversibility of the converter (introduced by I. Novikov) [7].

The reversible limit $\left(d_{I}=0\right)$ well restores the Carnot efficiency of the equilibrium Thermodynamics, according to a more coherent scheme than the one proposed by Wang and He [12] and conforming to previous definitions [13].

\subsection{Finite-time optimization of engine energy}

We will assume here that the 4 transformations of the cycle are done in finite time, according to the schema similar to those used in some works including [12]. It is thus assumed that each transformation takes place in a duration $\zeta_{i}$, but with a finite total duration $\zeta$ imposed for the cycle such that

$$
\sum_{i} \zeta_{i}=\zeta
$$

The simplest hypothesis for dissipations is, whatever the transformation, to assume them inversely proportional to $\zeta_{i}$ :

$$
\Delta S_{I i}=\frac{C_{I i}}{\zeta_{i}}
$$

with $C_{I i}$ irreversibility coefficients $[\mathrm{J} . \mathrm{s} / \mathrm{K}]$.

Indeed, this hypothesis well restores the quasi-static limit of the equilibrium Thermodynamics when $\zeta_{i} \rightarrow \infty$. It should be noted that the $C_{I i}$ irreversibility coefficients are positive finite quantities, having the dimension [J.s/K]. We call them entropy production action (a new concept analogous to energy action).

The combination of $(13,16)$ and the use of constraint (15) lead by using the variational calculation to the optimization of the transformation times of the cycle in relation to the mechanical energy produced:

$$
W_{\text {real }}=W_{r e v}-\left(T_{H S}-T_{C S}\right) \times \sum_{i}\left[\frac{C_{I i}}{\zeta_{i}}\right] .
$$

And to the following Lagrangian:

$$
L\left(\zeta_{i}\right)=W_{\text {rev }}-\Delta T_{S} \times \sum_{i} \frac{C_{I i}}{\zeta_{i}}-\lambda \times\left[\sum_{i} \zeta_{i}-\zeta\right]
$$

Hence, the expression of the corresponding maximum energy (first sequential optimum):

$$
\operatorname{MAX}_{1} W_{r e a l}=W_{r e v}-\frac{\Delta T_{S}}{\zeta} \times\left(\sum_{i} \sqrt{C_{I i}}\right)^{2}
$$

It is to be noted that this result is more general than the mechanical energy ;it is valid for all kinds of energy forms excepted the thermal energy only concerned by source and sink and efficiency at maximum energy output becomes:

$$
\eta\left(\operatorname{MAX}_{1} w\right)=\eta_{C}\left(1-\frac{\left(\sum_{i} \sqrt{C_{I i}}\right)^{2}}{\xi \Delta S}\right)
$$

with $\xi \Delta S$, cycle entropic transfer action.

\subsection{Case where the entropic action is a finite physical dimension}

We have shown in paragraph 2.3 that the maximum mechanical energy is associated with an optimal distribution of the durations of each transformation of the cycle, as long as the total duration (or cycle period) is fixed (finite time constraint).

This first maximum in energy (19) appears as a function of the entropic actions of each thermodynamic transformation. By imposing that entropic actions are also a finite physical dimension such as:

$$
\sum_{i} C_{I i}=C_{I T}
$$

The optimization by the variational calculation then leads to the equipartition of the entropic actions of each transformation:

$$
C_{I i}^{*}=\frac{C_{I T}}{4}
$$

$C_{I T}$, total entropic action distributed optimally over the cycle. It corresponds to the second maximum of energy $\mathrm{MAX}_{2} W_{\text {real }}$, obtained sequentially:

$$
\mathrm{MAX}_{2} W_{\text {real }}=W_{\text {rev }}-4 \frac{\Delta T_{S}}{\zeta} C_{I T}
$$

The conclusion of the previous optimization calculation is that the different mechanisms of entropy production (at least 2: irreversibility of isotherms and adiabatics) lead to an optimal distribution of irreversible transformation times in finite time, even for energy optimization.

In addition, the introduction of the concept of entropic action has allowed us to show that, in the presence of a 
finite constraint of entropic actions, energy optimization occurs at the equipartition of entropic actions of the 4 transformations of the cycle. This result should be compared with other previously discovered results including reference [14].

We will now consider the power of the thermomechanical engine.

\section{Power optimization of an endo-irréversible Carnot engine}

It should be noted here that, without distinguishing them, the literature reveals two formally different approaches. Thus the first works $[6,8]$ concerned systems in steady state or permanent dynamic conditions in the sense of the fluid mechanics, in which time does not occur explicitly. Thus many publications, developing the hypothesis of a stationary dynamic regime, are not explicitly related to the Thermodynamics in Finite Time (TTF).

The second approach corresponds to that of Section 2 and focuses on a cycle of period $\zeta$, approach similar to that of Curzon and Ahlborn [4], but completed as it will be illustrated below.

\subsection{Synthesis of energy optimization}

We recall here the essential result obtained in Section 2:

in Irreversible Thermodynamics the MAX $W$ of converted energy is obtained, at the minimum of entropy production $\Delta \mathrm{S}_{\mathrm{I}}$. Thus if $\mathrm{T}_{\mathrm{CS}}=\mathrm{T}_{0}$, the ambient temperature, we obtain the theorem of GOUY-STODOLA $[15,16]$. We renew that converted energy is more general than mechanical energy as what the case at the time of Carnot.

On the other hand, this energy optimization concerns only one cycle, and not the behaviour of a system in transient mode. This will be the subject of future work, which has already begun [17].

\subsection{Power optimization}

The previous synthesis is taken as the starting point for the optimization in average power over a cycle $\overline{\dot{W}}(W)$ (and not the instantaneous power). This average power is a function of $\zeta$, the cycle period, obtained from (23) in the form:

$$
\overline{\dot{W}}=\frac{W_{r e v}}{\zeta}-\frac{4 \Delta T_{S} C_{I T}}{\zeta^{2}} .
$$

It is easy to see that this power is cancelled for $\zeta$ tending towards infinity (Equilibrium. Thermodynamics), but also for $\zeta_{\text {lim }}$, limiting period such that:

$$
\zeta_{\text {lim }}=\frac{4 \Delta T_{S} C_{I T}}{W_{\text {rev }}}=\frac{4 C_{I T}}{\Delta S_{\text {rev }}}=\frac{4 C_{I T}}{\Delta S_{s}} .
$$

Between these two values, there is a period $\zeta^{*}$ (or a frequency $x^{*}$ ) leading to the maximum power:

$$
\zeta^{*}=\frac{8 \Delta T_{S} C_{I T}}{W_{\text {rev }}}=\frac{8 C_{I T}}{\Delta S_{s}} .
$$

This period is proportional to the total entropic action coefficient $C_{I T}$ and inversely proportional to the heat transfer entropy available at the hot source under reversibility conditions. Hence the maximum average power:

$$
\begin{aligned}
\operatorname{MAX} \overline{\dot{W}} & =\frac{1}{\zeta^{*}}\left[W_{\text {rev }}-\frac{4 \Delta T_{S} C_{I T}}{8 C_{I T}} \Delta S_{s}\right] \\
\operatorname{MAX} \overline{\dot{W}} & =\frac{\Delta S_{s}}{8 C_{I T}}\left[\Delta T_{S} \Delta S_{s}-\frac{\Delta T_{S} \Delta S_{s}}{2}\right] \\
& =\frac{\Delta T_{S} \Delta S_{s}{ }^{2}}{16 C_{I T}} .
\end{aligned}
$$

The efficiency associated with this MAX $\overline{\dot{W}}$ is:

$$
\eta_{I}(\operatorname{MAX} \overline{\dot{W}})=\frac{\eta_{C}}{16} \times \frac{\Delta S_{s}}{C_{I T}} \times \frac{8 C_{I T}}{\Delta S_{s}}=\frac{\eta_{C}}{2} .
$$

We therefore confirm a result that appears repeatedly in some works. The average maximum power output differs from the one presented by Curzon-Ahlborn. It is, according to us, more general since resulting from a triple sequential optimization of the Carnot endo-irreversible engine (energy optimisation in relation to $\zeta_{i}$; optimization in relation to the coefficients of action for the production of entropy of the transformations $C_{I I} ;$ average power optimization with respect to $\zeta$ cycle period).

\section{Discussion - synthesis of results}

This note, unlike most of the existing works, considers a thermomechanical engine model of exo-reversible and endo-irreversible Carnot cycle, while endo-reversibility is rather developed. In addition, if thermal losses are considered, it has been shown that the efficiency in the sense of the first law of thermodynamics depends on 2 ratios having as reference the heat transfer entropy available at the source under reversibility conditions:

- $d_{I}$, the degree of irreversibility of the converter.

$-d_{L S}$, the degree of thermal loss of the system including source and sink. This ratio characterizes the system's non-adiabaticity.

In the case of the endo-irreversible Carnot engine without thermal losses while retaining the same entropic reference, it has been shown that there is a first optimum of mechanized ( and more) energy related to the durations of isothermal and adiabatic transformations. This is to put in parallel to results reported in [14]. To do this, we introduced the new concept of entropic action for each transformation. We obtained an expression of the maximum energy depending on the duration of the cycle and the entropy production actions of each transformation.

A second optimization of the mechanized energy which presupposes the actions of production of entropy finished and bounded by a total entropy production action $C_{I T}$, leads to an optimal distribution with equipartition of the entropy production actions of each transformation.

The average power optimization of the endo-irreversible Carnot engine was then conducted in relation to the duration of the cycle. The optimum of this power is 
obtained for a period that is:

$$
\xi^{*}=\frac{8 C_{I T}}{\Delta S_{S}} .
$$

The optimal period is therefore related to the ratio of the total entropy production action of the cycle divided by the heat transfer entropy available in the reversible case at the heat source. The efficiency associated with the average maximum power is then half the Carnot efficiency, generally confirming a result that appears in some works. The result differs fundamentally from the one obtained by Curzon and Ahlborn in the endoreversible case.

Extensions of this proposal arein progress. They follow communications made in 2019 at two international conferences (JETC19, ECOS19).

This article was initially written in French in tribute to $\mathrm{S}$. Carnot, one of the fathers of thermodynamics, and probably the most cited French scientist in the world thanks to his opuscule [2], unique work of his short life.

\section{Author contribution statement}

M. Feidt realized the modeling. The results were discussed jointly by the two authors. The form of the paper was organized by R. Feidt. Improvements and comments to reviewers are done in common. - Full names of conferences (JETC19, ECOS19): JETC19, ECOS10 (not only acronyms). Joint european thermodynamics conference, Barcelona Spain, 21-24 May 2019. 32d International conference on efficiency, cost, optimization, simulation and environmental impact of energy systems, Wroclaw Poland, 23-28 June 2019.

\section{References}

1. L. Carnot, Principes fondamentaux de l'équilibre et du mouvement, Paris (1803)

2. S. Carnot, Réflexion sur la puissance motrice du feu, Paris, Bachelier (1824)

3. V.M. Brodiansky, Sadi Carnot 1796-1832, collection Etudes, Presses Universitaires de Perpignan (2006)

4. F. Curzon, B. Ahlborn, Am. J. Physiol. 43, 22 (1975)

5. M. Feidt, Entropy 19, 369 (2017)

6. P. Chambadal, Les centrales nucléaires (Armand Colin, Paris, France, 1957)

7. I. Novikov, At. Energ. 3, 409 (1957)

8. L. Ser, in Traité de physique industrielle: production et utilisation de la chaleur, pp. 848-852 (source gallica.bnf.fr) (1888-1892)

9. M. Feidt, M. Costea, S. Petrescu, C. Stanciu, Entropy 18, $243(2016)$

10. M. Blaise, M. Feidt, D. Maillet, Energy Convers. Manage. 163, $444(2018)$

11. J. Gonzales-Ayala, J.M. Roco, A. Medina, A. CalvoHernandez, Entropy 19, 182 (2017)

12. J. Wang, J. He, Phys. Rev. E 86, 051112 (2012)

13. R. Esposito, R. Kawai, K. Lindenberg, C. Vandenbroeck, Phys. Rev. Lett. 105, 150603 (2010)

14. D Tondeur, Optimisation thermodynamique, Equipartition de production d'entropie, chapitres BE8017, BE 8018 (15 pages, 19 pages), Techniques de l'ingénieur (2013)

15. I. Lucia, AAPP 94, 1 (2016)

16. M. Gouy, Journal de Physique. 94, 501 (1889)

17. M Feidt, in Finite Physical Dimension Optimal Thermodynamics, 1 Fundamentals (chapter 6, ISTE-ELSEVIER, 2017), pp. 189-191

Open Access This article is distributed under the terms of the Creative Commons Attribution License https://creativecom mons.org/licenses/by/4.0 which permits unrestricted use, distribution, and reproduction in any medium, provided the original author(s) and source are credited.

Cite this article as: Michel Feidt, Renaud Feidt, Endo-irreversible thermo-mechanical Carnot engine with new concept of entropy production action coefficient, Eur. Phys. J. Appl. Phys. 94, 30901 (2021) 